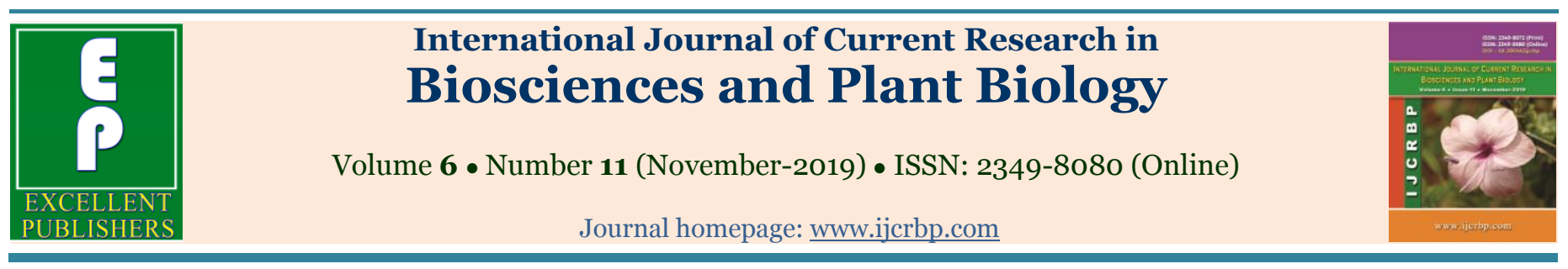

\title{
Combining ability studies in rabi sorghum by using line $\times$ tester analysis
}

\author{
Snehal A. Tambe ${ }^{* 1}$, D. V. Kusalkar ${ }^{2}$, G. C. Shinde ${ }^{1}$ and M. S. Shinde3 \\ ${ }^{1}$ Department of Botany, Mahatma Phule Krishi Vidyapeeth, Rahuri, Ahmednagar, Maharashtra - 413 722, India \\ ${ }^{2}$ Associate Director of Research ZARS, Igatpuri, MPKV, Rahuri, Ahmednagar, Maharashtra- 413 722, India \\ 3 All India Coordinated Sorghum Improvement Project, MPKV, Rahuri, Ahmednagar, Maharashtra- 413 722, India \\ *Corresponding author; e-mail: avinash.jondhale51@gmail.com
}

\begin{tabular}{|c|c|}
\hline Art & CT \\
\hline & \multirow{3}{*}{$\begin{array}{l}\text { Combining ability studies for grain yield and its components was studied using four } \\
\text { male sterile lines and twelve testers at Sorghum Improvement Project, MPKV, Rahuri, } \\
\text { Maharashtra. The mean squares due to lines, testers and lines x tester were highly } \\
\text { significant indicating the presence of variability for most of the characters. Estimates of } \\
\text { general combining ability (GCA) and specific combining ability (SCA) variances } \\
\text { indicated the predominance of non-additive gene action for most of the characters. The } \\
\text { line } 104 \text { A, RMS 2010-10A and testers RSR 986, RSR 1012, RSR 1019, RSR 1003 and } \\
\text { RSR 955 were observed good general combiner for grain yield and yield contributing } \\
\text { characters. The hybrid viz., 104A x RSR 1003, RMS 2010-10A x RSR 1013, RMS 2010- } \\
\text { 10A x RSR 955, 185A x RSR 1027, 185A x RSR 1020, RMS 2010-24A x RSR 1019, RMS } \\
\text { 2010-24A x RSR 1012, 185A x RSR 986, RMS 2010-24A x RSR 1020 and RMS 2010- } \\
\text { 24A x RSR 986 etc. exhibited with highly significant and positive SCA effects for grain } \\
\text { yield which could be exploited for development of hybrids. }\end{array}$} \\
\hline Ke」 & \\
\hline & \\
\hline
\end{tabular}

\section{Introduction}

Sorghum [Sorghum bicolor (L.) Moench] is an important cereal crop which is grown globally for food and fodder purpose. It is most widely grown in the semi-arid tropics where water availability is limited and frequently subjected to drought. India ranks first in terms of area under sorghum (5.82 M ha), while it ranks second in production i.e. 4.5 MT. The productivity of sorghum in India $(854 \mathrm{~kg}$ $\mathrm{ha}^{-1}$ ) is much less than the world average of $1457 \mathrm{~kg}$ ha $^{-1}$ (FAO, 2016). Combining ability studies provide useful information regarding the selection of suitable parents for effective hybridization program. It also provides information on various types of gene action involved in the expression of quantitative characters. It is also used for reducing the number of crosses to be advanced. Line $\times$ Tester analysis developed by Kempthorne (1957) and modified by Arunachalum (1974) appeared to be the most useful tool for screening large number of parental lines with rapidity and has practical utility in hybrid development breeding programme.

\section{Materials and methods}

Erect The present investigation was conducted at Sorghum Improvement Project, MPKV, Rahuri, Maharashtra during rabi2015-16. Four male sterile 
lines (185A, RMS2010-10A, RMS2010-24A, 104 A) were crossed with twelve testers (RSR 950, RSR 1012, RSR 1013, RSR 984, RSR 1014, RSR 1019, RSR 986, RSR 987, RSR 1020, RSR 1027, RSR 1003, RSR 955) in line $\times$ testers mating design used to develop $48 \mathrm{~F}_{1}$ 's hybrids. The resulting $48 \mathrm{~F}_{1}$ 's along with their 16 parents and one check ( $\mathrm{CSH}-15$ R) were evaluated for grain yield and yield contributing traits in rabi sorghum during 2015-16.

The parents and hybrids were grown in separate block in a Randomized Block Design (RBD) with two replications. Each entry was consisted two rows having $3 \mathrm{~m}$ length with inter and intra spacing at $45 \mathrm{~cm}$ and $15 \mathrm{~cm}$, respectively. Data were recorded on five randomly selected plants in each replication for the characters viz., days to $50 \%$ flowering, days to physiological maturity $(\mathrm{cm})$, plant height, 1000 grain weight $(\mathrm{g})$, dry fodder per plant, harvest index and grain yield per plant (g). Data were subjected to statistical analysis as per Arunachalam (1974) to understand the magnitude of general combining ability (GCA) effects of parents and specific combining ability (SCA) effects of F1's.

\section{Results and discussion}

The analysis of variance for combining ability (Table 2) revealed the presence of significant differences due to lines, testers and lines $\mathrm{x}$ testers for all the characters studied indicating the existence of variability among parents and hybrids. The testers contributed a major share of variance for all the characters. The estimates of components of variance for GCA were larger in magnitude than SCA for the characters days to 50\% flowering, days to physiological maturity and plant height indicating predominance of additive gene action. While the estimates of specific combining ability $\left(\sigma^{2}\right.$ SCA) variance were higher than general combining ability ( $\sigma^{2}$ GCA) variance for the characters 1000 grain weight, harvest index, dry fodder/plant and grain yield/plant indicates predominance of non-additive gene action (Jadhav and Deshmukh, 2017).

The combining ability helps in selection of potential parents and to know the magnitude of general and specific combining ability variances. The relative magnitude of GCA and SCA variances and GCA effects of parents and SCA effects of their crosses provides valuable guidelines for choice of parents and for planning suitable breeding methodology.

\section{Mean performance and General Combining Ability (GCA) Effects}

The mean performance and GCA effects of parents for yield and yield contributing traits in rabi sorghum are presented in Tables 1 and 3 . Estimates of GCA effects indicated that, none of the parents was a good general combiner for all the traits studied. The line 104 A showed most promising for all the studied characters. Among the line $185 \mathrm{~A}$ and from testers RSR 986 showed good general combining ability for days to flowering. The line $185 \mathrm{~A}, 104 \mathrm{~A}$ and tester RSR 986 also exhibited highly significant GCA in desirable direction for days to flowering and days to maturity. However, it showed negative GCA effect for grain yield per plant. The line RMS 201010 A and RMS 2010-24 A were bad combiners for days to maturity since they showed significant GCA effects in non-desirable direction. The lines RMS 2010-10 A and 104 A was showed significant positive GCA effects and hence it was best general combiner for grain yield per plant. While testers namely RSR 1012, RSR1019, RSR 1014 and RSR 955 were the best general combiners they showed higher magnitude of positive GCA effects for grain yield per plant. The GCA effects of parents and their per se performance indicated that the parent showing high mean performance also showed high general combining ability for grain yield per plant. However, 104 A was good combiner for physiological maturity, 1000 grain weight, plant height, dry fodder yield per plant and grain yield per plant (Jadhav and Deshmukh, 2017) and the female line 2010-10A was found good combiner for plant height, 1000 grain weight, harvest index and grain yield per plant.

As regards GCA effects of male parents, RSR 986 was good general combiner for days to 50 per cent flowering, days to physiological maturity and 1000 grain weight, RSR 1012 for plant height, grain yield per plant, dry fodder yield and harvest index while RSR 1019 was good general combiner for plant height and dry fodder yield, harvest index and grain yield per plant. 
Table 1. Mean performance of parents and hybrids for yield and yield contributing characters in rabi sorghum $(\mathrm{L} \times \mathrm{T})$.

\begin{tabular}{|c|c|c|c|c|c|c|c|c|}
\hline $\begin{array}{l}\text { Sr. } \\
\text { No. }\end{array}$ & $\begin{array}{l}\text { Name of parents/ } \\
\text { crosses }\end{array}$ & $\begin{array}{l}\text { Days to } \\
\mathbf{5 0 \%} \\
\text { flowering }\end{array}$ & $\begin{array}{l}\text { Days to } \\
\text { physic. } \\
\text { Maturi. }\end{array}$ & $\begin{array}{l}\text { Plant } \\
\text { height } \\
\text { (cm) }\end{array}$ & $\begin{array}{l}\text { 100o } \\
\text { grain } \\
\text { weight } \\
\text { (g) } \\
\end{array}$ & $\begin{array}{l}\text { Harvest } \\
\text { index (\%) }\end{array}$ & $\begin{array}{l}\text { Dry fodder } \\
\text { yield/plant } \\
\text { (g) }\end{array}$ & $\begin{array}{l}\text { Grain } \\
\text { yield/ } \\
\text { plant } \\
\text { (g) }\end{array}$ \\
\hline & Female (Lines) & & & & & & & \\
\hline 1 & $185 \mathrm{~A}$ & 73.8 & 114.1 & 119.1 & 25.9 & 20.5 & $75 \cdot 5$ & 21.5 \\
\hline 2 & RMS2010-10A & 80 & 120.2 & 112.3 & 21.99 & 33.9 & 47 & 22.2 \\
\hline 3 & RMS2010-24A & $75 \cdot 3$ & $115 \cdot 3$ & 122.3 & 25.2 & 28 & 40.7 & 27.2 \\
\hline \multirow[t]{2}{*}{4} & $104 \mathrm{~A}$ & 70 & 109.5 & $147 \cdot 3$ & 28 & 34.9 & 44.5 & 30.3 \\
\hline & $\begin{array}{l}\text { Lines mean } \\
\text { Male (Testers) }\end{array}$ & 74.8 & 114.8 & 125.3 & $\mathbf{2 5 . 2 7}$ & $\mathbf{2 9 . 3 3}$ & $\mathbf{5 1 . 9 3}$ & $25 \cdot 3$ \\
\hline 5 & RSR 950 & 88.5 & 124.9 & 121 & 29.3 & 34 & 49.5 & 46.2 \\
\hline 6 & RSR1012 & 88.4 & 128.2 & 176.5 & 25.13 & 33.6 & 47.5 & 50.2 \\
\hline 7 & RSR1013 & 87.1 & 127.2 & 146.3 & 27.7 & 31.2 & 41 & 33.2 \\
\hline 8 & RSR984 & 88.6 & 128.6 & 126.9 & 30.8 & 30.1 & 42.5 & 34.6 \\
\hline 9 & RSR1014 & 88.1 & 128.1 & 170.5 & $27 \cdot 3$ & 38.8 & 44.2 & 48.3 \\
\hline 10 & RSR1019 & 86.1 & 126.2 & 191.6 & 28.6 & 27.4 & 88.0 & 43.7 \\
\hline 11 & RSR986 & 63.7 & 104.1 & 132.8 & 24.1 & 21.4 & 70.0 & 26.9 \\
\hline 12 & RSR987 & 89.0 & 129 & 187.9 & 22.7 & 29.5 & $97 \cdot 3$ & 47.7 \\
\hline 13 & RSR1020 & 88.3 & 128.3 & 175.6 & 25.6 & 34.1 & 96.3 & 64.1 \\
\hline 14 & RSR1027 & 89.0 & 129.1 & 200.6 & 26.1 & 40.3 & 36.5 & 51.6 \\
\hline 15 & RSR1003 & 87.6 & 127.7 & 221.8 & 24.8 & 40.0 & 59.4 & 72.2 \\
\hline \multirow[t]{2}{*}{16} & RSR955 & 89.1 & 129.1 & 189.2 & 27.1 & 36.2 & 80.0 & 49.9 \\
\hline & $\begin{array}{l}\text { Testers mean } \\
\text { Hybrids }\end{array}$ & 86.13 & 125.9 & 170.1 & 26.6 & 33.05 & 62.68 & $47 \cdot 38$ \\
\hline 17 & 185 A x RSR 950 & 72.7 & 113.2 & 118.9 & $23 \cdot 3$ & 29.4 & 62.5 & 24.5 \\
\hline 18 & 185 A x RSR 1012 & 71.1 & 111.6 & 178.2 & $24 \cdot 3$ & $43 \cdot 3$ & 50.0 & 52.5 \\
\hline 19 & 185 A X RSR 1013 & 72.9 & 113.5 & 166.7 & 22.8 & 36.8 & 36.5 & 35.8 \\
\hline 20 & 185 A X RSR 984 & 65.9 & 107.6 & 154.9 & 22.9 & 32.2 & 36.5 & 30.0 \\
\hline 21 & 185 A X RSR 1014 & 74.1 & 114.6 & 155.0 & 22.7 & 26.4 & 43.5 & 31.5 \\
\hline 22 & 185AXRSR1019 & 73.6 & 113.6 & 194.9 & 24.5 & $33 \cdot 7$ & 94.0 & 54.5 \\
\hline 23 & 185AXRSR 986 & 64.5 & 105.0 & 180.1 & 27.2 & 26.9 & 103.5 & $37 \cdot 5$ \\
\hline 24 & 185AXRSR987 & $74 \cdot 3$ & 114.7 & 121.5 & 22.8 & 30.9 & 41.5 & 28.0 \\
\hline 25 & 185AXRSR1020 & 74.7 & $115 \cdot 5$ & 132.4 & 24.0 & 32.8 & 61.0 & 46.5 \\
\hline 26 & 185AXRSR1027 & 74.2 & 114.6 & 162.8 & 24.9 & 39.4 & 59.5 & 43.0 \\
\hline 27 & 185AXRSR1003 & 74.3 & 114.9 & 147.4 & 25.8 & 26.4 & 82.5 & 31.5 \\
\hline 28 & 185AXRSR 955 & 69.9 & 110.6 & 165.7 & 23.4 & 31.4 & 80.5 & 40.5 \\
\hline 29 & 2010-10AxRSR 950 & 72.6 & 112.9 & $155 \cdot 5$ & 23.9 & 36.1 & 52.5 & 39.5 \\
\hline 30 & 2010-10AxRSR1012 & 74.6 & 114.9 & 206.5 & $25 \cdot 7$ & 34.6 & 80.5 & 54.5 \\
\hline 31 & 2010-10AxRSR1013 & 75.1 & 115.2 & 182.9 & 28.0 & 40.5 & $77 \cdot 5$ & 66.9 \\
\hline 32 & 2010-10AxRSR 984 & 77.7 & 117.9 & 200.4 & 27.7 & 36.7 & 86.5 & 58.5 \\
\hline 33 & 2010-10AxRSR1014 & 77.0 & 117.5 & 169.5 & 24.5 & 42.2 & 53.5 & 63.5 \\
\hline 34 & 2010-10AxRSR1019 & 79.1 & 119.2 & 187.4 & 26.5 & 33.7 & 66.0 & 48.5 \\
\hline 35 & 2010-10AxRSR 986 & 67.8 & 108.6 & 175.7 & 29.8 & 30.7 & $55 \cdot 5$ & 35.0 \\
\hline 36 & 2010-10AxRSR 987 & 74.9 & 115.8 & 168.4 & 26.9 & 38.3 & $55 \cdot 5$ & $55 \cdot 5$ \\
\hline 37 & 2010-10AxRSR1020 & 75.2 & 115.6 & 131.8 & 23.6 & 36.9 & 49.0 & $45 \cdot 5$ \\
\hline 38 & 2010-10AxRSR1027 & 79.3 & 119.6 & 173.4 & 27.9 & 34.3 & $75 \cdot 5$ & 49.0 \\
\hline 39 & 2010-10AxRSR1003 & 79.0 & 119.4 & 185.5 & 24.9 & 37.4 & 49.5 & 44.5 \\
\hline 40 & 2010-10AxRSR955 & 73.9 & 114.4 & 182.8 & 31.2 & 35.8 & 78.5 & 69.0 \\
\hline 41 & 2010-24AxRSR950 & 81.2 & 121.7 & 140.4 & 22.6 & 33.6 & 41.5 & $37 \cdot 3$ \\
\hline 42 & 2010-24AxRSR1012 & 76.5 & 116.6 & 171.2 & 26.6 & 43.6 & 49.4 & 60.0 \\
\hline 43 & 2010-24AxRSR1013 & 80.5 & 121.1 & 160.2 & 22.5 & 41.5 & 35.0 & 41.0 \\
\hline 44 & 2010-24AxRSR984 & 82.5 & 122.9 & 136.9 & 24.5 & 33.0 & $45 \cdot 5$ & 37.5 \\
\hline 45 & 2010-24AxRSR1014 & 76.8 & 117.2 & 155.8 & 28.9 & 34.5 & 59.5 & $55 \cdot 5$ \\
\hline 46 & 2010-24AxRSR1019 & 81.5 & 121.8 & 192.3 & 25.5 & 36.3 & 67.5 & 47.0 \\
\hline 47 & 2010-24AxRSR 986 & 67.1 & 107.5 & 156.9 & 29.6 & 39.9 & 43.0 & 42.5 \\
\hline 48 & 2010-24AxRSR 987 & 82.4 & 123.0 & 122.0 & 26.9 & 35.6 & 58.5 & 44.5 \\
\hline 49 & 2010-24AxRSR1020 & 82.0 & 122.5 & 142.5 & 22.8 & 36.6 & 59.0 & 51.5 \\
\hline 50 & 2010-24AxRSR1027 & 82.2 & 122.0 & 111.3 & 24.7 & 31.9 & $37 \cdot 5$ & 32.5 \\
\hline 51 & 2010-24AxRSR1003 & 81.6 & 122.9 & 142.0 & 28.2 & $27 \cdot 5$ & 83.0 & 34.0 \\
\hline 52 & 2010-24AxRSR 955 & 81.9 & 122.1 & 155.6 & 24.6 & 27.8 & 86.5 & 38.5 \\
\hline
\end{tabular}


Table 1. Cntd....

\begin{tabular}{|c|c|c|c|c|c|c|c|c|}
\hline $\begin{array}{l}\text { Sr. } \\
\text { No. }\end{array}$ & $\begin{array}{l}\text { Name of parents/ } \\
\text { crosses }\end{array}$ & $\begin{array}{l}\text { Days to } \\
50 \% \\
\text { flowering }\end{array}$ & $\begin{array}{l}\text { Days to } \\
\text { physic. } \\
\text { Maturi. }\end{array}$ & $\begin{array}{l}\text { Plant } \\
\text { height } \\
\text { (cm) }\end{array}$ & $\begin{array}{l}\text { 100o } \\
\text { grain } \\
\text { weight } \\
\text { (g) }\end{array}$ & $\begin{array}{l}\text { Harvest } \\
\text { index }(\%)\end{array}$ & $\begin{array}{l}\text { Dry fodder } \\
\text { yield/plant } \\
\text { (g) }\end{array}$ & $\begin{array}{l}\text { Grain } \\
\text { yield/ } \\
\text { plant } \\
\text { (g) }\end{array}$ \\
\hline 53 & 104A x RSR 950 & 76.2 & 116.4 & 149.5 & 26.2 & 28.8 & 100.5 & 48.5 \\
\hline 54 & 104A x RSR 1012 & 70.5 & 110.5 & 200.6 & $25 \cdot 7$ & 36.2 & 94.0 & 76.5 \\
\hline 55 & 104A X RSR 1013 & 70.0 & 110.1 & 160.4 & 26.4 & 28.2 & 96.0 & 42.5 \\
\hline 56 & 104A X RSR 984 & 74.5 & $114 \cdot 7$ & 198.0 & 30.0 & 36.0 & 81.5 & 58.5 \\
\hline 57 & 104A X RSR 1014 & 75.6 & 116.0 & 190.6 & 26.7 & 31.0 & 102.5 & 60.0 \\
\hline 58 & 104A X RSR 1019 & 75.9 & 116.1 & 214.0 & 28.6 & 44.9 & 79.5 & 71.0 \\
\hline 59 & 104A X RSR 986 & 80.0 & 120.2 & 158.6 & 27.0 & 33.5 & 32.0 & 30.7 \\
\hline 60 & 104A X RSR 987 & $75 \cdot 3$ & $115 \cdot 3$ & 194.7 & 23.4 & 33.1 & 64.1 & 44.2 \\
\hline 61 & 104A X RSR 1020 & $75 \cdot 3$ & 115.6 & 164.1 & 27.7 & 37.2 & 40.5 & 31.1 \\
\hline 62 & 104 A X RSR 1027 & 74.7 & 114.7 & 172.9 & 26.4 & 32.7 & 63.5 & 33.5 \\
\hline 63 & 104 A X RSR 1003 & 74.8 & 175.2 & 207.4 & 24.6 & 39.9 & 70.5 & 70.0 \\
\hline 64 & 104 A X RSR 955 & 66.0 & 106.3 & 195.0 & 31.6 & 37.7 & 54.5 & 55.5 \\
\hline \multirow[t]{5}{*}{65} & CSH 15R (Ch) & 66 & 106.4 & 170.7 & 25.9 & 28.6 & 61.5 & 41 \\
\hline & Hybrid mean (HM) & 75.28 & 116.9 & 166.5 & 25.85 & $34 \cdot 75$ & 64.09 & 46.45 \\
\hline & $\mathbf{S E} \pm$ & $3 \cdot 39$ & 3.44 & 2.82 & 0.71 & 1.72 & 4.29 & 3.26 \\
\hline & CD at $5 \%$ & 6.82 & 6.92 & 5.68 & 1.42 & 3.46 & 8.63 & 6.55 \\
\hline & CD at $1 \%$ & 9.10 & 9.24 & 7.59 & 1.90 & 4.61 & 11.52 & 8.75 \\
\hline
\end{tabular}

Table 2. Analysis of variance for combining ability and estimates of GCA and SCA variances in rabi sorghum.

\begin{tabular}{|c|c|c|c|c|c|c|c|c|}
\hline Sources & D F & $\begin{array}{l}\text { Days to } \\
50 \% \\
\text { flowering }\end{array}$ & $\begin{array}{l}\text { Days to } \\
\text { physio. } \\
\text { maturity }\end{array}$ & $\begin{array}{l}\text { Plant } \\
\text { height } \\
(\mathrm{cm})\end{array}$ & $\begin{array}{l}1000 \\
\text { grain } \\
\text { weight (g) }\end{array}$ & $\begin{array}{l}\text { Harvest } \\
\text { index (\%) }\end{array}$ & $\begin{array}{l}\text { Dry fodder } \\
\text { yield per } \\
\text { plant(g) }\end{array}$ & $\begin{array}{l}\text { Grain } \\
\text { yield per } \\
\text { plant(g) }\end{array}$ \\
\hline Replication & 1 & 0.25 & 1.81 & 3.78 & 13.28 & 2.41 & 0.01 & 0.20 \\
\hline Treatments & 63 & $87.87^{* *}$ & $82.61^{* *}$ & $1548.62^{* *}$ & $10.95^{* *}$ & $51.03^{* *}$ & $819.62^{* *}$ & $349.40^{* *}$ \\
\hline Parents & 15 & $132.69^{* *}$ & $128.29^{* *}$ & $2371.15^{* *}$ & $10.89^{* *}$ & $68.49^{* *}$ & $900.13^{* *}$ & $432.41^{* *}$ \\
\hline Line & 3 & $34.21^{*}$ & $38.66^{*}$ & $478.79^{* *}$ & $12.62^{* *}$ & $87.79^{* *}$ & $507.44^{* *}$ & $35.11^{*}$ \\
\hline Testers & 11 & $101.30^{* *}$ & $97.19^{* *}$ & $2013.74^{* *}$ & $10.48^{* *}$ & $60.12^{* *}$ & $1025.92^{* *}$ & $314.08 * *$ \\
\hline Line vs.Tester & 1 & $773.44^{* *}$ & $739.26^{* *}$ & $11979.60^{* *}$ & $10.26^{* *}$ & $102.61^{* *}$ & $694.45^{* *}$ & $2926.04^{* *}$ \\
\hline Parent vs.hybrid & 1 & $1540.24^{* *}$ & $1321.65^{* *}$ & $1393.85^{* *}$ & $3 \cdot 37^{*}$ & $129.62^{* *}$ & $403.44^{* *}$ & $537.23^{* *}$ \\
\hline Hybrids & 47 & $42.66^{* *}$ & $41.68^{* *}$ & $1289.41^{* *}$ & 11.12 & $43.79^{* *}$ & $802.78^{* *}$ & $318.91^{* *}$ \\
\hline Error & 63 & 10.36 & 10.83 & 6.86 & 0.50 & 3.19 & 16.50 & 9.24 \\
\hline \multicolumn{9}{|l|}{ Estimates } \\
\hline$\sigma^{2} \mathrm{GCA}$ & & 8.81 & 8.66 & 273.61 & 1.61 & 3.07 & 53.37 & 50.83 \\
\hline$\sigma^{2} \mathrm{SCA}$ & & 5.49 & 4.87 & 238.64 & 3.59 & 20.29 & 426.17 & 95.02 \\
\hline$\sigma^{2} \mathrm{~A}$ & & 17.62 & 17.32 & 547.23 & 3.22 & 6.14 & 107.34 & 101.67 \\
\hline$\sigma^{2} \mathrm{D}$ & & 5.49 & 4.86 & 238.64 & 3.59 & 20.29 & 426.17 & 95.02 \\
\hline$\sigma^{2} \mathrm{~A} / \sigma^{2} \mathrm{D}$ & & 3.21 & 3.56 & 2.29 & 0.89 & 0.30 & 0.25 & 1.07 \\
\hline
\end{tabular}

RSR 1003 for plant height and dry fodder yield, whereas RSR 955 was good general combiners for plant height, 1000 grain weight, dry fodder yield and grain yield per plant. (Table.3).These studies would help the selection of parents for the incorporation of the specific characters in the new progeny (Kale, 2012). The studies on general combining ability effects indicates that the parents showing good general combining ability for grain yield also possess either average or good general combining abilities for two or more yield contributing characters.

\section{Specific Combining Ability (SCA) effects}

Specific combining ability effects are indicative of heterosis. Similarly, they represent both dominant and epistatic gene actions. The two cross combinations viz., 185A x RSR 984 (good x average) and104 A x RSR 955 (average x average) were displayed significant negative SCA effects for days to 50 per cent flowering. For physiological maturity highly significant SCA effects in the desirable direction were observed in the crosses viz; 104A x RSR 955 (average x average), 185A x 
RSR 984 (good x average) indicating the presence of dominance or non-allelic interactions (Prabhakar et al., 2013).

For plant height, twenty-two cross combinations were showed significant positive SCA effects. The crosses, 104A x RSR 987 (good x poor), 185A x RSR 986 (poor $x$ average), 104A x RSR 1003 (good $x$ good), RMS 2010-10A x RSR 984 (good x good), RMS 2010-10A x RSR 1027 (good x poor), RMS 2010-10A x RSR 1020 (good x poor), RMS 201024A x RSR 950 (poor x poor), RMS 2010-24A x RSR 1019 (poor x good), RMS 2010-24A x RSR 1013 (poor $x$ average) and 185 A x RSR 1013 (poor x average) were showed higher magnitude of positive SCA effects (Kumar and Chand, 2015).

For 1000 grain weight, seventeen cross combinations were showed significant positive SCA effects. The crosses RMS 2010-24A x RSR 1014 (average $x$ average), RMS 2010-24A x RSR 1003 (average $x$ average), 104A x RSR 984 (good x average) and 104A x RSR 955 (good x good),RMS 2010-10A x RSR 1012(good $x$ average), RMS 201024A x RSR 987(average x poor),RMS 2010-10A x RSR 955(average $x$ good),104A x RSR 1020(good $x$ poor),185A x RSR 1003(poor $x$ average), were expressed significant positive SCA effects (Samdur et al.,2013).

For harvest index, out of forty-eight crosses, twelve cross combinations 104A x RSR 1019 (average x good), 185A x RSR 1027 (poor $x$ average), RMS 2010-10A x RSR 1014 (good x poor), 104A x RSR 1003 (average $x$ poor), RMS 2010-24A x RSR 986 (good x poor), 185A x RSR 1012 (poor x good), RMS 2010-10A x RSR 1019 (good x good), 104A x RSR 955 (average $x$ poor) and RMS 2010-24A x RSR 1013(good $x$ poor), were showed high magnitude of positive SCA effects (Jadhav and Deshmukh, 2017).

For dry fodder yield per plant, eighteen cross combinations were showed significant positive SCA effects. The crosses, 185A x RSR 986 (average $\mathrm{x}$ poor), 104A $\mathrm{x}$ RSR 1014 (good $\mathrm{x}$ average), 104A x RSR 950 (good $x$ average), 104A x RSR 1013 (good $\mathrm{x}$ average), RMS 2010-10A x RSR 984 (average $\mathrm{x}$ average), RMS 2010-24A x RSR 1003 (poor $x$ good), RMS 2010-24A x RSR 955 (poor $\times$ good) and 185 A x RSR 1019 (average $x$ good) were showed high magnitude of positive SCA effects (Dehinwal et al., 2017).

Table 3. General combining ability effects of parents for yield and yield contributing characters in rabi sorghum.

\begin{tabular}{|c|c|c|c|c|c|c|c|c|}
\hline $\begin{array}{l}\text { Sr. } \\
\text { No }\end{array}$ & Parents & $\begin{array}{l}\text { Days to } \\
50 \% \\
\text { flowering }\end{array}$ & $\begin{array}{l}\text { Days to } \\
\text { physiological } \\
\text { maturity }\end{array}$ & $\begin{array}{l}\text { Plant } \\
\text { height } \\
\text { (cm) }\end{array}$ & $\begin{array}{l}\text { 100o } \\
\text { grain } \\
\text { weight } \\
\text { (g) }\end{array}$ & $\begin{array}{l}\text { Harvest } \\
\text { index(\%) }\end{array}$ & $\begin{array}{l}\text { Dry fodder } \\
\text { yield per } \\
\text { plant(g) }\end{array}$ & $\begin{array}{l}\text { Grain } \\
\text { yield per } \\
\text { plant(g) }\end{array}$ \\
\hline & Female & & & & & & & \\
\hline 1 & $185 \mathrm{~A}$ & $-3.43^{* *}$ & $-3.25^{* *}$ & $-9.94^{* *}$ & $-1.67^{* *}$ & $-2.27^{* *}$ & -1.47 & $-8.610^{* *}$ \\
\hline 2 & RMS2010-10A & 0.24 & 0.24 & $10.17^{* *}$ & $0.83^{* *}$ & $1.69^{* *}$ & 0.91 & $5.90^{* *}$ \\
\hline 3 & RMS2010-24A & $4.40^{* *}$ & $4.43^{* *}$ & $-17.56^{* *}$ & -0.28 & 0.39 & $-8.60^{* *}$ & $-3.11^{* *}$ \\
\hline \multirow[t]{2}{*}{4} & $104 \mathrm{~A}$ & -1.213 & $-1.42^{*}$ & $17.33^{* *}$ & $1.12^{* *}$ & 0.20 & $9.17^{* *}$ & $5.82^{* *}$ \\
\hline & $\begin{array}{l}\text { SE士 } \\
\text { Males }\end{array}$ & 0.69 & 0.70 & 0.58 & 0.14 & 10.35 & 0.88 & 0.67 \\
\hline 5 & RSR950 & 0.396 & 0.37 & $-25.41^{* *}$ & $-1.91^{* *}$ & $-2.78^{* *}$ & 0.16 & $-9.14^{*}$ \\
\hline 6 & RSR 1012 & -2.10 & -2.28 & $22.64^{* *}$ & -0.30 & $4.69^{* *}$ & $4 \cdot 38^{* *}$ & $14.28^{* *}$ \\
\hline 7 & RSR1013 & -0.65 & -0.70 & 1.07 & $-0.98^{* *}$ & $2.03^{* *}$ & -2.84 & 0.21 \\
\hline 8 & RSR 984 & -0.13 & 0.096 & $6.07^{* *}$ & 0.41 & -0.23 & -1.59 & -0.47 \\
\hline 9 & RSR 1014 & 0.60 & 0.65 & 1.24 & -0.21 & $-1.24^{*}$ & 0.66 & $6.031^{* *}$ \\
\hline 10 & RSR 1019 & 2.25 & 1.99 & $30.67^{* *}$ & 0.36 & $2.38^{* *}$ & $12.66^{* *}$ & $8.66^{* *}$ \\
\hline 11 & RSR 986 & $-5.43^{* *}$ & $-5 \cdot 35^{* *}$ & 1.34 & $2.50^{* *}$ & $-2.04^{* *}$ & $-5.59^{* *}$ & $-10.17^{* *}$ \\
\hline 12 & RSR 987 & 1.45 & 1.52 & $-14.83^{* *}$ & $-0.89^{* *}$ & -0.28 & $-9.19^{* *}$ & $-3.54^{* *}$ \\
\hline 13 & RSR 1020 & 1.52 & 1.57 & $-23.78^{* *}$ & $-1.38^{* *}$ & -1.12 & $-11.72^{* *}$ & $-1.44^{*}$ \\
\hline 14 & RSR 1027 & 2.32 & 2.05 & $-11.38^{* *}$ & 0.10 & -0.08 & $-5.09 * *$ & $-7.09^{* *}$ \\
\hline 15 & RSR 1003 & 2.15 & 2.42 & $4.09^{* *}$ & 0.003 & $-1.98^{* *}$ & $7.28^{* *}$ & -1.59 \\
\hline \multirow[t]{2}{*}{16} & RSR 955 & -2.35 & -2.33 & $8.29^{* *}$ & $2.29^{* *}$ & $-1.60^{* *}$ & $10.91^{* *}$ & $4.28^{* *}$ \\
\hline & $\mathbf{S E} \pm$ & 1.20 & 1.22 & 0.99 & 0.25 & 0.61 & 1.52 & 1.15 \\
\hline
\end{tabular}

\footnotetext{
* Significant at $5 \%$ level; ** Significant at $1 \%$ level.
} 
Table 4. Specific combining ability (SCA) effects for yield and yield contributing characters in 48 crosses of rabi sorghum.

\begin{tabular}{|c|c|c|c|c|c|c|c|c|}
\hline $\begin{array}{l}\text { Sr. } \\
\text { No. }\end{array}$ & Crosses & $\begin{array}{l}\text { Days to } \\
\mathbf{5 0 \%} \\
\text { flowering }\end{array}$ & $\begin{array}{l}\text { Days to } \\
\text { physiological } \\
\text { maturity }\end{array}$ & $\begin{array}{l}\text { Plant } \\
\text { height } \\
\text { (cm) }\end{array}$ & $\begin{array}{l}\text { 100o } \\
\text { grain } \\
\text { weight } \\
\text { (g) }\end{array}$ & $\begin{array}{l}\text { Harvest } \\
\text { index (\%) }\end{array}$ & $\begin{array}{l}\text { Dry fodder } \\
\text { yield per } \\
\text { plant(g) }\end{array}$ & $\begin{array}{l}\text { Grain yield } \\
\text { per } \\
\text { plant(g) }\end{array}$ \\
\hline 1 & $1 \mathrm{X}_{5}$ & 0.45 & 0.40 & $-12.23^{* *}$ & 0.96 & -0.32 & -0.28 & -4.34 \\
\hline 2 & $1 \mathrm{X} 6$ & 1.35 & 1.45 & -0.98 & 0.44 & $6.14^{* *}$ & $-17.01^{* *}$ & 0.24 \\
\hline 3 & $1 X_{7}$ & 1.70 & 1.77 & $9.09^{* *}$ & 0.39 & 2.34 & $-23.28^{* *}$ & $-2.39^{*}$ \\
\hline 4 & 1X8 & $-5.82^{*}$ & $-4.92^{* *}$ & $-7.71^{* *}$ & $-1.68 * *$ & -0.02 & $-24.53^{* *}$ & $-7.515^{* *}$ \\
\hline 5 & $1 X_{9}$ & 1.65 & 1.52 & -2.78 & $-1.31^{*}$ & $-4.88^{* *}$ & $-19.78^{* *}$ & $-12.52^{* *}$ \\
\hline 6 & $1 \mathrm{X} 10$ & -0.50 & -0.83 & $7.69^{* *}$ & -0.11 & -1.16 & $18.72^{* *}$ & $7.86^{*}$ \\
\hline 7 & $1 \mathrm{X} 11$ & -1.92 & -2.08 & $22.22^{* *}$ & 0.44 & $-3.60^{*}$ & $46.47^{* *}$ & $9.69^{* *}$ \\
\hline 8 & $1 \mathrm{X} 12$ & 1.00 & 0.75 & $-20.21^{* *}$ & -0.56 & -1.34 & $-11.93^{* *}$ & $-6.44^{* *}$ \\
\hline 9 & $1 \mathrm{X} 13$ & 1.33 & 1.30 & -0.36 & $1.15^{*}$ & -0.79 & $10.09^{* *}$ & $9.96^{* *}$ \\
\hline 10 & $1 \mathrm{X} 14$ & 0.03 & 0.12 & $17.64^{* *}$ & 0.65 & $7 \cdot 30^{* *}$ & 1.97 & $12.11^{* *}$ \\
\hline 11 & 1X15 & 0.30 & 0.05 & $-13.23^{* *}$ & $1.52^{* * *}$ & $-4.13^{* *}$ & $12.59^{* *}$ & $-4.89^{*}$ \\
\hline 12 & $1 \mathrm{X} 16$ & 0.40 & 0.50 & 0.87 & $-1.13^{*}$ & 0.47 & $6.97^{* *}$ & -1.78 \\
\hline 13 & $2 X_{5}$ & $-3 \cdot 31$ & -3.39 & $4.26^{*}$ & -0.85 & $2.47^{*}$ & $-12.66^{* *}$ & -3.85 \\
\hline 14 & $2 \mathrm{X} 6$ & 1.19 & 1.26 & $7.21^{* *}$ & -0.72 & $-6.51^{* *}$ & $11.12^{* *}$ & $-12.27^{* *}$ \\
\hline 15 & $2 X_{7}$ & 0.24 & -0.01 & $5.18^{*}$ & $2.28^{* *}$ & 2.07 & $15 \cdot 34^{* *}$ & $14.20^{* *}$ \\
\hline 16 & $2 \times 8$ & 2.31 & 1.89 & $17.68^{* *}$ & 0.57 & 0.53 & $23.09^{* *}$ & $6.48^{* *}$ \\
\hline 17 & $2 \times 9$ & 0.89 & 0.94 & $-8.39^{* *}$ & $-2.04^{* *}$ & $6.97^{* *}$ & $-12.16^{* *}$ & $4.98^{*}$ \\
\hline 18 & $2 X 10$ & 1.34 & 1.29 & $-19.92^{* *}$ & -0.58 & $5.17^{* *}$ & $-11.66^{* *}$ & $-12.65^{* *}$ \\
\hline 19 & $2 \mathrm{X} 11$ & -2.29 & -1.96 & -2.29 & 0.57 & $-3.70^{* *}$ & -3.91 & $-7.32^{* *}$ \\
\hline 20 & $2 \mathrm{X} 12$ & -2.06 & -1.64 & $6.58^{* *}$ & $1.03^{*}$ & 2.18 & -0.31 & $6.55^{* *}$ \\
\hline 21 & $2 \mathrm{X} 13$ & -1.84 & -1.89 & $-21.07^{* *}$ & $-1.75^{* *}$ & -0.65 & -4.28 & $-5.55^{*}$ \\
\hline 22 & $2 X 14$ & 1.46 & 1.64 & $8.13^{* *}$ & $1.10^{*}$ & -2.0 & $15.59^{* *}$ & 3.60 \\
\hline 23 & $2 \mathrm{X} 15$ & 1.34 & 1.06 & $4.76^{*}$ & $-1.75^{* *}$ & $2.91^{*}$ & $-22.78 * *$ & $-6.40^{* *}$ \\
\hline 24 & $2 \mathrm{X} 16$ & 0.74 & 0.81 & -2.14 & $2.13^{*}$ & 0.91 & 2.59 & $12.23^{* *}$ \\
\hline 25 & $3 \times 5$ & 1.12 & 1.22 & $16.88^{* *}$ & $-1.15^{*}$ & 1.27 & $-14.15^{* * *}$ & 2.96 \\
\hline 26 & $3 \times 6$ & -1.08 & -1.23 & -0.37 & $1.32^{*}$ & $3.75^{* *}$ & $-10.47^{* *}$ & 2.34 \\
\hline 27 & $3 X_{7}$ & 1.47 & 1.70 & $10.21^{* *}$ & $-2.19^{* *}$ & $4 \cdot 36^{*}$ & $-17.65^{* *}$ & -2.69 \\
\hline 28 & $3 \times 8$ & 2.95 & 2.70 & $-18.09^{* *}$ & $-1.51^{* *}$ & -1.94 & $-8.40^{* *}$ & $-5.52^{*}$ \\
\hline 29 & $3 \times 9$ & -3.48 & -3.55 & $5.63^{* *}$ & $3.44^{* *}$ & 0.60 & $3 \cdot 35$ & $5.99^{*}$ \\
\hline 30 & $3 \times 10$ & -0.43 & -0.30 & $12.71^{* *}$ & -0.50 & -1.20 & -0.65 & $-5.14^{*}$ \\
\hline 31 & $3 \mathrm{X} 11$ & -7.15 & -7.25 & $6.63^{* *}$ & $1.50^{* *}$ & $6.75^{* *}$ & -6.90 & $9.19^{* *}$ \\
\hline 32 & $3 \mathrm{X} 12$ & 1.27 & 1.37 & $-12.09^{* *}$ & $2.26^{* *}$ & 0.72 & $12.20^{* *}$ & 4.56 \\
\hline 33 & $3 \mathrm{X} 13$ & 0.80 & 0.82 & $17 \cdot 36^{* *}$ & $-1.41^{* *}$ & 0.33 & $15.23^{* *}$ & $9.46^{* *}$ \\
\hline 34 & $3 \mathrm{X} 14$ & 0.20 & -0.15 & $-26.24^{* *}$ & $-1.05^{*}$ & $-3.16^{*}$ & $-12.90^{* *}$ & -3.89 \\
\hline 35 & $3 \mathrm{X} 15$ & -0.23 & 0.37 & $-11.02^{* *}$ & $2.61^{* *}$ & $-5.73^{* *}$ & $20.23^{* *}$ & $-7.89^{* *}$ \\
\hline 36 & $3 \times 16$ & 4.57 & 4.32 & -1.62 & $-3 \cdot 33^{-}$ & $-5.75^{* *}$ & $20.10^{* *}$ & $-9.27^{* *}$ \\
\hline 37 & $4 X_{5}$ & 1.74 & 1.77 & $-8.91^{* *}$ & $1.04^{*}$ & $-3.42^{*}$ & $27.09^{* *}$ & 5.23 \\
\hline 38 & $4 \mathrm{X} 6$ & -1.46 & -1.48 & $-5.86 * *$ & $1.04^{*}$ & $-3 \cdot 38^{*}$ & $16.36^{* *}$ & $9.80^{* *}$ \\
\hline 39 & $4 X_{7}$ & -3.41 & -3.45 & $-24.48^{* *}$ & 0.30 & $-8.76^{*}$ & $25.59^{* *}$ & $-9.12^{* *}$ \\
\hline 40 & $4 \times 8$ & 0.56 & 0.35 & $8.12^{* *}$ & $2.61^{* *}$ & 1.43 & $9.84^{*}$ & $6.55^{* *}$ \\
\hline 41 & $4 \times 9$ & 0.94 & 1.10 & $5.54^{* *}$ & -0.09 & $-2.68 *$ & $28.59^{* *}$ & 1.55 \\
\hline 42 & $4 \times 10$ & -0.41 & -0.15 & -0.48 & $1.18^{*}$ & $7.53^{* *}$ & $-6.42^{*}$ & $9.93^{* *}$ \\
\hline 43 & $4 \mathrm{X} 11$ & $11.36^{* *}$ & 11.30 & $-26.56^{* *}$ & $-2.52^{* *}$ & 0.55 & $-35.67^{* *}$ & $-11.55^{* *}$ \\
\hline 44 & $4 \mathrm{X} 12$ & -0.21 & -0.48 & $25.72^{* *}$ & $-2.72^{* *}$ & -1.55 & 0.04 & $-4.67^{*}$ \\
\hline 45 & $4 X 13$ & -0.29 & -0.23 & $4.07^{*}$ & $2.09^{* *}$ & 1.11 & $-21.04^{* *}$ & $-13.67^{* *}$ \\
\hline 46 & $4 X 14$ & -1.69 & -1.60 & 0.47 & -0.71 & -2.14 & $-4.67^{*}$ & $-11.82^{* *}$ \\
\hline 47 & $4 \mathrm{X} 15$ & -1.41 & -1.48 & $19.49^{* *}$ & $-2.39^{* *}$ & $6.94^{* *}$ & $-10.04^{* *}$ & $19.18^{* *}$ \\
\hline 48 & $4 \mathrm{X} 16$ & $-5.71^{*}$ & -5.63 & 2.89 & $2.33^{* *}$ & $4.37^{* *}$ & $-29.66^{* *}$ & -1.20 \\
\hline & $\mathbf{S E} \pm$ & 2.40 & 2.43 & 1.99 & 0.50 & 1.22 & 3.03 & 2.30 \\
\hline & ant at & $\mathrm{el} ;{ }^{* *} \mathrm{Si}$ & $\mathrm{t}$ at $1 \%$ level. & & & & & \\
\hline Parer & $1.105^{2}$ & & $10-10 \mathrm{~A}$ & & & o & 7. 1 & \\
\hline & 8. RSH & 984 & 10. RSI & 11. & 986 & 13. RS & 14. RS & 27 \\
\hline & 15. RS & R 1003 & & & & & & \\
\hline
\end{tabular}


Nineteen cross combinations were showed significant positive SCA effects for grain yield per plant. The crosses 104A x RSR 1003 (good x poor), RMS 2010-10A x RSR 1013 (good $x$ average), RMS 2010-10A x RSR 955 (good x good), 185A x RSR 1027 (poor x poor), 185A x RSR 1020 (poor $\mathrm{x}$ poor), RMS 2010-24A x RSR 1019 (poor x good), RMS 2010-24A x RSR 1012 (poor x good), 185A x RSR 986 (poor $x$ poor), RMS 2010-24A x RSR 1020 (poor x poor) and RMS 2010-24A x RSR 986 (poor $\mathrm{x}$ poor)were showed high magnitude of positive SCA effects (Chaudhary et al., 2006; Premalatha et al., 2006; Kalpande et al., 2019). These crosses also showed high SCA effects for one or more yield contributing characters. When the crosses showing good SCA effects involves either both or one parent with poor/average GCA effects indicating prevalence of non-additive gene action.

The higher magnitude of SCA variance over GCA variance was observed in 1000 grain weight, harvest index, dry fodder/plant and grain yield/plant which indicate the importance of nonadditive gene action for these characters (Samdur et al., 2013). The overall gene action studies from combining ability analysis indicated that dominance or non-additive gene action was important for most of the characters studied.

\section{Conflict of interest statement}

Authors declare that they have no conflict of interest.

\section{References}

Arunachalam, V., 1974. The fallacy behind the use of modified Line $\mathrm{x}$ tester design, India. Indian J. Genet. 34(2), 280-287.

Chaudhary, S.B., Patil, J. V., Thombare, B.B., Kulkarni, V.M., 2006. Selection of parents based on combining ability studies in sorghum [Sorghum bicolor (L.) Moench]. Ann. Plant Physiol. 20(1), 95-97.
Dehinwal, A. K., Pahuja1, S. K., Shafiqurrahaman, M., Anil Kumar, Sharma, P., 2017. Studies on combining ability for yield and its component traits in forage sorghum. Int. J. Pure App. Biosci. 5(5), 493-502.

FAO, 2016. Production Yearbook. FAO, Rome, Italy.

Jadhav, R. R., Deshmukh, D. T., 2017. Heterosis and combining ability studies in sorghum (Sorghum bicolor (L.) Moench) over the environments. Int. J. Curr. Microbiol. Appl. Sci. 6(10), 3058-3064.

Kale, B. H., 2012. Genetic and stability analysis for yield and yield contributing traits over different seasons in sorghum [Sorghum bicolor (L.) Moench]. Doctoral (Ph.D.) Thesis submitted to Navsari Agricultural University, Navsari.

Kalpande, H.V., Ranjana Tiwari., Kalyankar, S.V., 2019. Heterosis studies for yield, it's component and grain mould resistance in sorghum genotype. J. Pharmacogn. Phytochem. 8(3), 2907-2914.

Kempthorne, O., 1957. An introduction to genetic statistics. John Willey and Sons, London. pp 208-221.

Kumar, S., Chand, P., 2015. Combining ability and heterosis for grain yield, fodder yield and other agronomic traits in sorghum [Sorghum bicolor (L.) Moench]. Appl. Nat. Sci. Found. 7, 2.

Prabhakar, Elangovan, M., Bahadure, D.M., 2013. Combining ability of new parental lines for flowering, maturity and grain yield in rabi sorghum. Electronic J. Plant Breed. 4(3), 12411218.

Premalatha, N., Kumaravadivel, Veerabadhiran, P., 2006. Heterosis and combining ability for grain yield and its components in sorghum (Sorghum bicolor (L.) Moench). Indian J. Genet. 66(2), 123-126.

Samdur, M. Y., Prabhakar, Hariprasanna, K., Mukesh, P., Patil, J. V., 2013. Combining ability studies in rabi sorghum [Sorghum bicolor (L.) Moench]. Conference: Global Consultation on Millets Promotion for Health \& Nutritional Security, DSR, Hyderabad,p.334.

\section{How to cite this article:}

Tambe, S. A., Kusalkar, D. V., Shinde, G. C., Shinde, M. S., 2019. Combining ability studies in rabi sorghum by using line $\times$ tester analysis. Int. J. Curr. Res. Biosci. Plant Biol. 6(11), 22-28.

doi: https://doi.org/10.20546/ijcrbp.2019.611.003 